\title{
ON ZERO-ONE LAWS
}

\author{
JOHN C. MORGAN II
}

AbSTRaCt. An extension of a topological zero-one law due to $M$. and K.P.S. Bhaskara Rao and of the Hewitt-Savage zero-one law is presented.

In [8] M. and K.P.S. Bhaskara Rao proved the following topological zeroone law, which we shall refer to as the Bhaskara Rao zero-one law.

THEOREM 1. Let $X$ be a topological space and let $\Phi$ be a group of homeomorphisms of $X$ onto itself with the property that for any two nonempty open sets $U$ and $V$, there exists a homeomorphism $\varphi \in \Phi$ such that $\varphi(U) \cap V \neq \varnothing$. If $S \subset X$ is a set with the classical Baire property such that $\varphi(S)=S$ for all $\varphi \in \Phi$, then either $S$ or $X-S$ is of the first category.

This theorem was first stated by Oxtoby [7] for a complete separable metric space without isolated points and a cyclic group; a proof in the case of a complete metric space and any countable group was given in [2, p. 75]. A theorem very closely related to that of [8] was also found, independently of $\mathbf{M}$. and K.P.S. Bhaskara Rao, by Kuratowski (see [5, Theorem 1]); in fact by a slight modification of the proof of the latter theorem, one easily obtains the implication $(1) \rightarrow(4)$ of [8].

Using Theorem 1, M. and K.P.S. Bhaskara Rao obtained a category analogue of the Hewitt-Savage zero-one law. The analogy here is, however, incomplete, since the measure analogue of their category analogue is not the Hewitt-Savage zero-one law itself, but an extension of this theorem to symmetric sets measurable with respect to the completion of a product probability space.

In this paper an abstract zero-one law is established which provides a further extension of the Bhaskara Rao zero-one law and also furnishes the proper measure-theoretic analogue.

1. Terminology. It has been shown in [6] that several analogies between category and measure can be unified within an abstract theory of Baire category starting with the notion of a $\Omega$-family. In this section we recall some basic definitions and facts from [6] which are pertinent to this paper.

Received by the editors February 18, 1976 and, in revised form, July 7, 1976.

AMS (MOS) subject classifications (1970). Primary 54A05, 60F20; Secondary 28A05.

Key words and phrases. Zero-one laws, invariant sets, Baire property. 
Whenever $\mathbb{Q}$ is a family of sets, we shall refer to the members of $\mathscr{Q}$ as $\mathbb{Q}$-sets.

Definition 1. A family $\mathcal{C}$ of subsets of a set $X$ is called a $\Re$-family if the following axioms are satisfied.

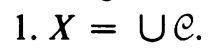

2. Let $A$ be a $C$-set and let $\mathscr{D}$ be a nonempty family of disjoint $C$-sets which has power less than the power of $\mathcal{C}$. If $A \cap(\cup \mathcal{D})$ contains a $\mathcal{C}$-set, then there is a $D$-set $D$ such that $A \cap D$ contains a $C$-set. On the other hand, if $A \cap(\cup \mathscr{D})$ contains no -set, then there is a -set $B \subset A$ which is disjoint from all $\mathscr{D}$-sets.

The symbol $\mathcal{C}$ will always be used below to denote a $\Re$-family, with respect to which we define the generalized Baire category concepts.

Definition 2. A set $S \subset X$ is $\mathcal{C}$-singular if each $\mathcal{C}$-set $A$ contains a $C$-set $B$ which is disjoint from $S$. We denote by $\bigodot_{1}$ the family of all countable unions

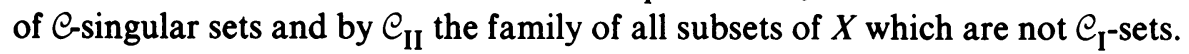

A set $S \subset X$ is a $C_{\mathrm{II}^{-}}$-set everywhere on a $C_{\text {set }} A$ if for every $C_{-s e t} B \subset A$, the set $S \cap B$ is a $\bigodot_{I^{-}}$-set. $S$ is a $\bigodot_{I^{-}}$-set everywhere if it is a $C_{I^{-}}$-set everywhere on every $C_{\text {-set; }}$ or, equivalently, if $B \cap S$ is a $\mathcal{C}_{\mathrm{II}}$-set for every $\mathcal{C}_{\text {-set }} B$.

The following theorem is a generalization of a theorem of Banach (see [6, Theorem 2]).

FUndamental THEOREM. If $S$ is a $\mathcal{C}_{\mathrm{II}^{-}}$set, then $S$ is a $\mathrm{C}_{\mathrm{II}^{-}}$-set everywhere on some C-set.

Definition 3. A set $S \subset X$ has the Baire property with respect to $C$ if for every $C$-set $A$ there is a $C$-set $B \subset A$ such that either $B \cap S$ or $B \cap(X-S)$ is a $\mathcal{C}_{\mathrm{I}}$-set.

We shall denote the family of subsets of $X$ which have the Baire property with respect to $e$ by $\mathfrak{B}(\mathcal{C})$.

As is easily seen, $\mathfrak{B}(\mathcal{C})$ is a $\sigma$-field containing the $\sigma$-ideal of $C_{1}$-sets and the family $C$.

\section{An abstract zero-one law.}

Definition 4. Let $X$ be a nonempty set and let $\Phi$ be a set of one-to-one mappings of $X$ onto itself. A family $\mathcal{S}$ of subsets of $X$ is invariant under $\Phi$, or $\Phi$-invariant, if $\varphi(\delta)=\delta$ for all $\varphi \in \Phi$. A set $S \subset X$ is $\Phi$-invariant if the family $\delta=\{S\}$ is $\Phi$-invariant; or, equivalently, if $\varphi(S)=S$ for all $\varphi \in \Phi$. Whenever $\Phi$ consists of a single mapping $\varphi$ we use the terminology "invariant under $\varphi$ " in lieu of " $\Phi$-invariant".

If $\mathcal{C}$ is a $\Omega$-family of subsets of $X$, then a set $S \subset X$ is called $C$-almost invariant under $\Phi$ if the symmetric difference $S \Delta \varphi(S)$ is a $C_{\mathrm{I}}$-set for all $\varphi \in \Phi$.

The following elementary lemma will be utilized in the proof of Theorem 2 .

LEMMA 1. If $\varphi$ is a one-to-one mapping of a set $X$ onto itself and $\mathcal{C}$ is a $\Re$-family of subsets of $X$ which is invariant under $\varphi$, then

(i) the families $\mathcal{C}_{\mathrm{I}}, \mathcal{C}_{\mathrm{II}}$, and $\mathfrak{B}(\mathcal{C})$ are invariant under $\varphi$. 
(ii) $A$ set $S \subset X$ is a $\mathcal{C}_{\mathrm{II}^{-}}$-set everywhere on a $\mathrm{C}_{\text {-set } A} A$ if and only if $\varphi(S)$ is a $\mathcal{C}_{\mathrm{II}^{-}}$-set everywhere on $\varphi(A)$.

THEOREM 2. Let $\Phi$ be a group of one-to-one mappings of a set $X$ onto itself, let $\mathcal{C}$ be a $\Re$-family of subsets of $X$ which is $\Phi$-invariant, and assume the following condition holds:

for all $A, B \in \mathcal{B}$ there is a mapping $\varphi \in \Phi$

such that $\varphi(A) \cap B$ contains $a$ C-set.

If $S \in \mathfrak{B}(\mathcal{C})$ is $\mathcal{C}$-almost invariant under $\Phi$, then either $S$ or $X-S$ is a $\mathcal{C}_{\mathrm{I}^{-}}$-set.

Proof. Assume $S$ is a $C_{I^{-}}$-set. By the Fundamental Theorem, $S$ is a $C_{I^{-}}$-set everywhere on some $C_{\text {-set }} A$. We show $S$ is a $C_{11}$-set everywhere.

Let $B$ be any $\mathcal{C}_{\text {-set, let }} \varphi \in \Phi$ satisfy condition (*), and let $C \subset \varphi(A) \cap B$ be a -set. According to Lemma $1, \varphi(S)$ is a $C_{\mathrm{II}}$-set everywhere on $\varphi(A)$, and hence $C \cap \varphi(S)$ is a $\bigodot_{I^{-}}$-set. The set $T=S \Delta \varphi(S)$ being a $C_{I^{-}}$set, $C \cap T$ is a $\mathcal{C}_{\mathrm{I}^{-}}$-set and $C \cap \varphi(S)-C \cap T$ is a $\mathcal{C}_{\mathrm{II}^{-}}$-set. From the inclusions $C \cap \varphi(S)$ $-C \cap T \subset C \cap S \subset B \cap S$ we conclude $B \cap S$ is a $\mathcal{C}_{\mathrm{II}}$-set for every $\mathcal{C}_{\text {-set }}$ B.

Since $S$ has the Baire property with respect to $C$ and is a $C_{\mathrm{II}}$-set everywhere, it follows from the Fundamental Theorem that $X-S$ is a $e_{\mathrm{I}^{-}}$-set.

3. The Bhaskara Rao zero-one law. When $X$ is a topological space, $C$ consists of all nonempty open sets, and $\Phi$ is a group of homeomorphisms of $X$ onto $X$, condition (*) is equivalent to

$$
\begin{aligned}
& \text { for any two nonempty open sets } U \text { and } V \text {, there exists } \\
& \text { a homeomorphism } \varphi \in \Phi \text { such that } \varphi(U) \cap V \neq \varnothing \text {. }
\end{aligned}
$$

Theorem 2 thus provides the following simple extension of Theorem 1.

Corollary 1. Let $X$ be a topological space, let $\Phi$ be a group of homeomorphisms of $X$ onto $X$ and assume condition (**) holds. If $S$ is a set with the classical Baire property such that $S \triangle \varphi(S)$ is of the first category for every $\varphi \in \Phi$, then either $S$ or $X-S$ is of the first category.

4. An extension of the Hewitt-Savage zero-one law. Let $(X, \overline{\mathscr{Y}}, \bar{P})$ be the completion of an infinite product probability space $(X, \mathscr{F}, P)$ in which all coordinate spaces are identical and $\mathscr{F}$ is the $\sigma$-field generated by the finitedimensional measurable rectangles. Throughout this section, $\Phi$ will denote the group of all one-to-one mappings of $X$ onto itself which permute only finitely many coordinates and leave the remaining coordinates unchanged.

According to the Hewitt-Savage zero-one law ([3, Theorem 11.3]; see also $[1, \S 8.1])$, if $S \in \mathscr{F}$ is $\Phi$-invariant, then $P(S)=0$ or $P(S)=1$. The category analogue of this theorem given in [8] states that if $X$ is an infinite product topological space in which all coordinate spaces are identical, then every $\Phi$ invariant subset of $X$ having the Baire property with respect to the product topology is either of the first category or its complement is of the first category. 
The appropriate measure analogue of this topological theorem is the statement: If $S \in \overline{\bar{F}}$ is $\Phi$-invariant, then $\bar{P}(S)=0$ or $\bar{P}(S)=1$. We shall now establish a stronger measure-theoretic result, whose category analogue is a consequence of Corollary 1.

COROllary 2. If $S \in \overline{\mathscr{F}}$ and $S \triangle \varphi(S)$ is of $\bar{P}$-probability zero for every $\varphi \in \Phi$, then $S$ has $\bar{P}$-probability zero or one.

Proof. As noted in [6], the family $\mathcal{C}$ of all sets in $\mathscr{F}$ of positive probability is a $\Omega$-family, the $\mathcal{C}_{\mathrm{I}}$-sets coincide with the sets of $\bar{P}$-probability zero, and $\mathfrak{B}(\mathcal{C})$ coincides with $\overline{\mathscr{F}}$. It is easily seen that $\mathscr{F}$ is $\Phi$-invariant. Also $P$ is $\Phi$-invariant; i.e., $P[\varphi(A)]=P(A)$ for all $A \in \mathscr{F}$ and all $\varphi \in \Phi$ (cf. [1, Theorem 8.1.3]). Therefore $\mathcal{C}$ is $\Phi$-invariant. It thus remains only to show condition (*) holds.

Suppose $A, B \in \mathcal{C}$. Since the $\sigma$-field $\mathscr{F}$ is generated by the field of all finitedimensional cylinder sets we can choose finite-dimensional cylinder sets $M, N$ so that $P(A \triangle M)<\frac{1}{4} P(A) P(B)$ and $P(B \triangle N)<\frac{1}{4} P(A) P(B)$. Assume $M$ is determined by the coordinates $i_{1}, \ldots, i_{m}$ and $N$ is determined by the coordinates $j_{1}, \ldots, j_{n}$. Let $\varphi \in \Phi$ replace the coordinates $i_{1}, \ldots, i_{m}$ with coordinates all distinct from $j_{1}, \ldots, j_{n}$. The set $\varphi(M) \cap N$ is then a finitedimensional cylinder set with

$$
P[\varphi(M) \cap N]=P[\varphi(M)] P(N)=P(M) P(N) .
$$

Now,

$$
P(M) \geqslant P(A \cap M)=P[A-(A-M)]=P(A)-P(A-M)
$$

and

$$
P(A-M) \leqslant P(A \triangle M)<\frac{1}{4} P(A) P(B) \leqslant \frac{1}{4} P(A) .
$$

Hence $P(M) \geqslant P(A)-\frac{1}{4} P(A)=\frac{3}{4} P(A)$. Similarly, $P(N) \geqslant \frac{3}{4} P(B)$. Therefore

$$
P[\varphi(M) \cap N]=P(M) P(N) \geqslant \frac{9}{16} P(A) P(B) .
$$

From the equality

$$
P[\varphi(M) \cap N]=P[\varphi(M) \cap N \cap \varphi(A)]+P[\varphi(M) \cap N-\varphi(A)]
$$

and the fact that

$$
P[\varphi(M) \cap N-\varphi(A)] \leqslant P[\varphi(M)-\varphi(A)]=P(M-A)<\frac{1}{4} P(A) P(B),
$$

we have

$$
\begin{aligned}
P[\varphi(A) \cap N] & \geqslant P[\varphi(M) \cap N \cap \varphi(A)] \geqslant\left(\frac{9}{16}-\frac{1}{4}\right) P(A) P(B) \\
& >\frac{1}{4} P(A) P(B)>P(N-B) \geqslant P[\varphi(A) \cap N-B] .
\end{aligned}
$$

But, since $P[\varphi(A) \cap N]=P[\varphi(A) \cap N \cap B]+P[\varphi(A) \cap N-B]$, we have 
$P[\varphi(A) \cap N \cap B]>0$ and, consequently, $P[\varphi(A) \cap B]>0$. Therefore $\varphi(A)$ $\cap B$ is a -set.

REMARKS. (1) If we take $e$ to be the family of all nonempty finitedimensional measurable rectangles, or the family of all nonempty finitedimensional cylinder sets, then $C$ is a $\Omega$-family and condition (*) is easily verified. Hence we may conclude from Theorem 2 that every set in $\mathfrak{B}(\mathcal{C})$ which is $C_{\text {-almost invariant under }} \Phi$ is either a $C_{\mathrm{I}}$-set or its complement is a $C_{\mathrm{I}}$-set. In general, however, the $\mathcal{C}_{\mathrm{I}}$-sets need not be of $\bar{P}$-probability zero. For example, let $X=\prod_{k=1}^{\infty} X_{k}$ where $X_{k}=\{1,2,3, \ldots\}$ for each $k$ and the probability measure $P_{k}$ on $X_{k}$ is defined by $P_{k}(1)=1 / 2, P_{k}(2)=1 / 4, \ldots, P_{k}(n)=1 / 2^{n}$, $\ldots$. The set $S=\prod_{k=1}^{\infty}\{1, \ldots, k\}$ is then -singular, but

$$
P(S)=\prod_{k=1}^{\infty}\left(1-1 / 2^{k}\right)>0 .
$$

Also, the sets of $\bar{P}$-probability zero are not always $\mathcal{C}_{\mathrm{I}}$-sets. This is easily seen by taking $X=\prod_{k=1}^{\infty} X_{k}$ where each $X_{k}$ is the same continuous probability space, taking a point $p$ in $X_{1}$, and considering the measurable rectangle $S=\{p\} \times \prod_{k=2}^{\infty} X_{k}$.

(2) Corollary 2 may be readily extended to include certain situations in which the coordinate spaces are not necessarily identical; see [4].

(3) Let $(X, \overline{\mathcal{F}}, \bar{P})$ be the completion of an infinite product probability space $(X, \mathscr{F}, P)$ in which all coordinate spaces are the real line $R$ with a fixed probability measure on the Borel subsets of $R$, and $\mathscr{F}$ is the $\sigma$-field generated by the finite-dimensional Borel cylinder sets. If $E$ is a linear analytic set, then we may apply Corollary 2 to show that the set of all elements $x=\left\langle x_{n}\right\rangle_{n=1}^{\infty}$ in $X$ whose partial sums $s_{n}=x_{1}+\cdots+x_{n}$ belong to $E$ infinitely often has $\bar{P}$ probability 0 or 1 . (Cf. [3, p. 493] and [1, p. 258] where the same result is obtained when $E$ is a linear Borel set.)

(4) The $\sigma$-field of "almost permutable" sets in [1, p. 253], is contained in the $\sigma$-field of sets which are -almost invariant under $\Phi$, but is generally smaller, as is easily seen from the example in the preceding remark where these $\sigma$-fields have different cardinalities.

(5) Concerning the existence of nonmeasurable $\Phi$-invariant sets, see [9].

ACKNOWLEDGMENT. The author is grateful to the referee for a substantial improvement of the original version of Theorem 2 above.

\section{REFERENCES}

1. K. L. Chung, $A$ course in probability theory, 2nd ed., Academic Press, New York, 1974.

2. W. H. Gottschalk and G. A. Hedlund, Topological dynamics, Amer. Math. Soc. Colloq. Publ., vol. 36, Amer. Math. Soc., Providence, R.I., 1955. MR 17, 650.

3. E. Hewitt and L. J. Savage, Symmetric measures on cartesian products, Trans. Amer. Math. Soc. 80 (1955), 470-501. MR 17, 863.

4. S. Horn and S. Schach, An extension of the Hewitt-Savage zero-one law, Ann. Math. Statist. 41 (1970), 2130-2131. MR 42 \# 3821. (See also Zbl. Math. 222 (1972), No. 60019.)

5. K. Kuratowski, On the concept of strongly transitive systems in topology, Ann. Mat. Pura Appl. (4) 98 (1974), 357-363. MR 49 \#9824. 
6. J. C. Morgan II, Baire category from an abstract viewpoint, Fund. Math. 94 (1976).

7. J. C. Oxtoby, Note on transitive transformations, Proc. Nat. Acad. Sci. U.S.A. 23 (1937), 443-446.

8. M. Bhaskara Rao and K.P.S. Bhaskara Rao, A category analogue of the Hewitt-Savage zeroone law, Proc. Amer. Math. Soc. 44 (1974), 497-499. MR 49 \#9823.

9. J. Rosenthal, Nonmeasurable invariant sets, Amer. Math. Monthly 82 (1975), 488-491. MR 51 \#3389.

Department of Mathematics, Syracuse University, Syracuse, New York 13210

Current address: Department of Mathematics, California State Polytechnic University, Pomona, California 91768 